\title{
Free Vibrations of Unsymmetrically Laminated Anisotropic Plates with Clamped Edges*
}

\author{
Charles W. Bert and Byron L. Mayberry $\uparrow$ \\ School of Aerospace and Mechanical Engineering \\ University of Oklahoma \\ Norman, Oklahoma 73069
}

\begin{abstract}
A linear analysis is presented for determining the natural frequencies of vibration of laminated anisotropic rectangular plates. The plate may consist of an arbitrary number of thin orthotropic layers, the major material-symmetry axis of each layer oriented arbitrarily with respect to the longer plate edge. An approximate solution is obtained by the Rayleigh-Ritz energy method. Numerical results are presented for fully clamped boundary conditions and compared with experimental results for symmetrically and unsymmetrically laminated plates.
\end{abstract}

\section{INTRODUCTION}

$\mathrm{T}$ HE IMPORTANCE of predicting plate or panel vibration characteristics has grown steadily in the past few years. Because of the critical strength/weight ratio in design, there is considerable interest in composite materials. Of the many kinds of composite configurations, fiberreinforced materials are most popular. On a macroscopic basis, such a composite behaves as if it were a homogeneous, anisotropic material, i.e. one that exhibits different elastic properties when tested along different directional orientations within the body. The particular kind of anisotropy exhibited by fiber-reinforced composites is that of an orthotropic material, which has three mutually perpendicular planes of elastic symmetry. When orthotropic layers oriented at arbitrary angular orientations are laminated to make a multi-layer structure, the structure is called laminated anisotropic and a coupling effect is produced. This coupling effect is due to the interaction of the stretching and the flexural effects.

"The research reported here is based on an unpublished thesis submitted by the second author in partial fulfillment of the requirements for the degree of Master of Engineering (Aerospace) at the University of Oklahoma, June 1968.

tPresently 2nd Lt., U. S. Air Force, Pilot Training, Webb AFB, Texas. 
Of the numerous analyses of vibrations in rectangular, specially orthotropic $^{*}$ plates referenced in a recent survey paper by Leissa [1], only eight considered fully clamped edges. Reference [2] is an additional analysis of this problem not referenced in [1].

Vibrations of generally anisotropic plates have been analyzed by Waddoups and Ashton [3-5]. Using the Rayleigh-Ritz approach, their analyses were formulated to use the characteristic beam functions tabulated in [6]. However, they compared with experimental results only for the completely free and cantilever cases. Later they compared results for the fully clamped case [7].

So far as arbitrarily laminated plates (including coupling) are concerned, References [8] and [9] considered layered isotropic material only. Stavsky [10] derived the differential equations, which included the coupling effect and anisotropic behavior, but did not obtain a solution for the coupled case. In his survey [1], Leissa mentioned recent work by Whitney in which closed-form solutions were obtained for arbitrarily laminated anisotropic plates for two simple boundary conditions.

A complete analysis is developed here for the small-deflection free vibration of laminated anisotropic rectangular plates and applied specifically to the practically important case of fully clamped edges. This is believed to be the first such analysis applicable to these boundary conditions.

\section{THEORETICAL VIBRATION ANALYSIS}

Using the theory of arbitrarily laminated plates obeying the Kirchhoff hypothesis, such as presented in [11], the following constitutive relation is obtained:

$$
\left\{\begin{array}{l}
N_{i} \\
M_{i}
\end{array}\right\}=\left[\begin{array}{ll}
A_{i j} & B_{i j} \\
B_{i j} & D_{i j}
\end{array}\right]\left\{\begin{array}{l}
\bar{\epsilon}_{j} \\
\kappa_{j}
\end{array}\right\} \quad(i, j=1,2,6)
$$

The equations for the elements of the stiffness matrices $A, B$, and $D$ are given below.

$$
\begin{aligned}
& A_{i j}=\sum_{k=1}^{N} \int_{-h / 2}^{h / 2} Q_{i j}^{(k)} d z=\sum_{k=1}^{N}\left(h_{k}-h_{k-1}\right) Q_{i j}^{(k)} \\
& B_{i j}=\sum_{k=1}^{N} \int_{-h / 2}^{h / 2} z Q_{i j}^{(k)} d z=\sum_{k=1}^{N} \frac{1}{2}\left(h_{k}^{2}-h_{k-1}^{2}\right) Q_{i j}^{(k)} \\
& D_{i j}=\sum_{k=1}^{N} \int_{-h / 2}^{h / 2} z^{2} Q_{i j}^{(k)} d z=\sum_{k=1}^{N} \frac{1}{3}\left(h_{k}^{3}-h_{k-1}^{3}\right) Q_{i j}^{(k)}
\end{aligned}
$$

\footnotetext{
"Onthotropic material with the axes of material symmetry aligned parallel to the respective edges of the plate.
} 
where $k$ refers to the $k$ th ply, $N$ is the total number of plies, $z$ is the thickness coordinate measured from the midplane of the plate, $h$ is the plate thickness, $h_{k}$ is the upper surface of the $k$ th ply, and $Q_{i j}$ is the $k$ th ply reduced stiffness coefficient, which can be related to the ply orientation $\theta_{k}$ and the elastic constants of the orthotropic ply [12].

Neglecting energy dissipation (damping), Hamilton's principle requires that the time integral of the difference between the potential and kinetic energies attain a stationary value. However, assuming that the displacements are proportional to $\sin \omega t$, the velocities are proportional to $\cos \omega t$. Since the integral of $\sin ^{2} \omega t$ is equal to the integral of $\cos ^{2} \omega t$, the time dependencies in the potential and kinetic energies cancel each other. Thus, it is convenient to apply Hamilton's principle in terms of the energies with the time dependencies eliminated, i.e. the amplitudes of the energies, $V$ and $T$. Thus, the following energy difference is defined:

$$
L=V-T
$$

Neglecting transverse shear and intralaminar shear effects, the potential energy amplitude can be written as follows:

$$
V=(1 / 2) \int_{0}^{a} \int_{0}^{b}\left(N_{1} \bar{\epsilon}_{1}+N_{2} \bar{\epsilon}_{2}+N_{6} \bar{\epsilon}_{6}+M_{1} \kappa_{1}+M_{2} \kappa_{2}+M_{6} \kappa_{6}\right) d y d x
$$

where the $N_{i}, \bar{\epsilon}_{j}, M_{i}, \kappa_{j}$ are functions of $x$ and $y$ only.

Substituting the values of the $N_{i}$ and $M_{i}$ from Equation (1) into Equation (4) would result in an expression for the potential energy in terms of the strains and curvatures.

Using the classical linear theory of small deflections of thin plates, two hypotheses will be used to convert the strains and curvatures to displacements. The hypotheses are 1) that displacements are infinitesimal, so that the linearized strain-displacement relations may be used; and 2) that slopes are sufficiently small, so that the linearized curvature expressions are adequate. Thus, the following relations for curvature and midplane strain hold:

$$
\begin{gathered}
\bar{\epsilon}_{1}=u,_{x} ; \bar{\epsilon}_{2}=v,_{y} ; \bar{\epsilon}_{6}=u,_{y}+v,_{x} \\
\kappa_{1}=-w,_{x x} ; \kappa_{2}=-\bar{w},_{y y} ; \kappa_{6}=-2 w,,_{x y}
\end{gathered}
$$

where $u$ and $v$ are the displacements in the $x$ and $y$ directions, $w$ is the normal deflection, and a comma denotes differentiation with respect to the variable following the comma.

Substituting Equations (1), (5), and (6) into Equation (4) gives the following expression for the potential energy amplitude in terms of the stiffness coefficients and displacements: 
Free Vibrations of Unsymmetrically Laminated Anisotropic Plates

$$
\begin{aligned}
& V=(1 / 2) \int_{0}^{a} \int_{0}^{b}\left[A_{11} u_{, x}^{2}+2 A_{12} u,,_{x} v,_{y}+2 A_{16}\left(u,,_{x}\right)\left(u,_{y}+v,,_{x}\right)+A_{22} v_{, y}^{2}\right. \\
& +2 A_{26}\left(v,{ }_{y}\right)\left(u, y+v,{ }_{x}\right)+A_{66}\left(u, y+v,{ }^{2}\right)^{2}-2 B_{11} u,{ }_{x} w, x x \\
& -2 B_{12} u,{ }_{x} w,_{y y}-2 B_{12} v,_{y} w,_{x x}-2 B_{22} v{ }_{, y} w,_{y y}-4 B_{16} u,_{x} w,_{x y} \\
& -2 B_{16}\left(w,{ }_{x x}\right)\left(u,,_{y}+v,{ }_{x}\right)-4 B_{26} v,_{y} w,_{x y}-2 B_{26}\left(w,_{y y}\right)\left(u,{ }_{y}+v,{ }_{x}\right) \\
& -4 B_{66}\left(w, x_{x y}\right)\left(u,{ }_{y}+v,_{x}\right)+D_{11} w^{2},_{x x}+2 D_{12} w_{, x x} w_{, y y}+4 D_{16} w,_{x x} w,_{x y} \\
& \left.+D_{22} w_{, y y}^{2}+4 D_{26} w,_{y y} w_{, x y}+4 D_{66} w_{, x y}^{2}\right] d y d x
\end{aligned}
$$

Neglecting rotatory inertia, the kinetic energy amplitude is written as follows:

$$
T=(1 / 2) \int_{0}^{a} \int_{0}^{b} \rho h \bar{v}^{2} d y d x
$$

where $\rho h$ is the mass per unit area, and $\bar{v}$ is the resultant velocity amplitude given by:

$$
\bar{v}^{2}=\omega^{2}\left(u^{2}+v^{2}+w^{2}\right)
$$

To apply the Rayleigh-Ritz method, the assumed displacement modal functions for the displacement amplitudes are given the following general notation:

$$
\begin{aligned}
w & =\sum_{n} \sum_{m} E_{n m} \phi_{w n}(\alpha) \phi_{w m}(\beta) \\
u & =\sum_{n} \sum_{m} F_{n m} \phi_{u n}(\alpha) \phi_{u m}(\beta) \\
v & =\sum_{n} \sum_{m} G_{n m} \phi_{v n}(\alpha) \phi_{v m}(\beta)
\end{aligned}
$$

where the $\phi$ 's are modal functions and $\alpha$ and $\beta$ denote the normalized arguments in the $x$ and $y$ directions, respectively, i.e. $x / a$ and $y / b$.

Substituting Equations (7-9) and (10) into Equation (3) yields the following result:

$$
\begin{aligned}
L & =(1 / 2) \sum_{n} \sum_{m}\left\{\int _ { 0 } ^ { 1 } \int _ { 0 } ^ { 1 } \left[A_{11}\left(F_{n m} \phi_{u n}^{\prime} \phi_{u m}\right)^{2}+2 A_{12}\left(G_{n m} \phi_{u n} \phi_{u m}^{\prime}\right)\left(F_{n m} \phi_{u n}^{\prime} \phi_{u m}\right)\right.\right. \\
& +A_{22}\left(G_{n m} \phi_{v n} \phi_{v m}^{\prime}\right)^{2}+2 A_{16}\left(F_{n m} \phi_{u n} \phi_{u m}^{\prime}+G_{n m} \phi_{u n}^{\prime} \phi_{v m}\right)\left(F_{n m} \phi_{u n}^{\prime} \phi_{u m}\right) \\
& +2 A_{26}\left(F_{n m} \phi_{u n} \phi_{u m}^{\prime}+G_{n m} \phi_{v n}^{\prime} \phi_{v m}\right)\left(G_{n m} \phi_{v n} \phi_{v m}^{\prime}\right)+A_{66}\left(F_{n m} \phi_{u n} \phi_{u m}^{\prime}\right. \\
& \left.+G_{n m} \phi_{v n}^{\prime} \phi_{v m}\right)^{2}-2 B_{11}\left(F_{n m} \phi_{u n}^{\prime} \phi_{u m}\right)\left(E_{n m} \phi_{w n}^{\prime \prime} \phi_{w m}\right) \\
& -B_{12}\left\{2\left(E_{n m} \phi_{w n}^{\prime \prime} \phi_{u m}\right)\left(G_{n m} \phi_{v n} \phi_{v m}^{\prime}\right)+2\left(E_{n m} \phi_{w n} \phi_{u m}^{\prime \prime}\right)\left(F_{n m} \phi_{u n}^{\prime} \phi_{u m}\right)\right\} \\
& -2 B_{22}\left(E_{n m} \phi_{w n} \phi_{w m}^{\prime \prime}\right)\left(G_{n m} \phi_{v n} \phi_{v m}^{\prime}\right)-B_{16}\left\{4\left(E_{n m} \phi_{w n}^{\prime} \phi_{u m}^{\prime}\right)\left(F_{n m} \phi_{u n}^{\prime} \phi_{u m}\right)\right.
\end{aligned}
$$




$$
\begin{aligned}
& \left.+2\left(F_{n m} \phi_{u n} \phi_{u m}^{\prime}+G_{n m} \phi_{v n}^{\prime} \phi_{v m}\right)\left(E_{n m} \phi_{i n n}^{\prime \prime} \phi_{w m}\right)\right\} \\
& -B_{26}\left\{4\left(E_{n m} \phi_{w n}^{\prime} \phi_{w m}^{\prime}\right)\left(G_{n m} \phi_{v n} \phi_{v m}^{\prime}\right)+2\left(F_{n m} \phi_{u n} \phi_{u m}^{\prime}\right.\right. \\
& \left.\left.+G_{n m} \phi_{v n}^{\prime} \phi_{v m}\right)\left(E_{n m} \phi_{w n} \phi_{w m}^{\prime \prime}\right)\right\}-4 B_{66}\left(F_{n m} \phi_{u n} \phi_{u m}^{\prime}\right. \\
& \left.+G_{n m} \phi_{v n}^{\prime} \phi_{v m}\right)\left(E_{n m} \phi_{w n}^{\prime} \phi_{w m}^{\prime}\right)+D_{11}\left(E_{n m} \phi_{w n}^{\prime \prime} \phi_{w m}\right)^{2} \\
& +2 D_{12}\left(E_{n m} \phi_{w n} \phi_{w m}^{\prime \prime}\right)\left(E_{n m} \phi_{w n}^{\prime \prime} \phi_{w m}\right)+D_{22}\left(E_{n m} \phi_{w n} \phi_{u m}^{\prime \prime}\right)^{2} \\
& +4 D_{16}\left(E_{n m} \phi_{w n}^{\prime} \phi_{u m}^{\prime}\right)\left(E_{n m} \phi_{w n}^{\prime \prime} \phi_{w m}\right)+4 D_{26}\left(E_{n m} \phi_{w n}^{\prime} \phi_{w m}^{\prime}\right)\left(E_{n m} \phi_{w n} \phi_{w m}^{\prime \prime}\right) \\
& \left.+4 D_{66}\left(E_{n m} \phi_{w m}^{\prime} \phi_{w m}^{\prime}\right)^{2}\right] d \alpha d \beta-\frac{\rho h \omega^{2}}{2} \sum_{n} \sum_{m} \int_{0}^{1} \int_{0}^{1}\left[\left(F_{n m} \phi_{u n} \phi_{u m}\right)^{2}\right. \\
& \left.+\left(G_{n m} \phi_{v n} \phi_{v m}\right)^{2}+\left(E_{n m} \phi_{w n} \phi_{w m}\right)^{2}\right] d \alpha d \beta
\end{aligned}
$$

where $\phi_{n}^{\prime}=\partial \phi_{n} / \partial \alpha, \phi_{m}^{\prime}=\partial \phi_{m} / \partial \beta$, etc.

At this point in the solution for the natural frequencies and modal shapes, the Rayleigh-Ritz method is applied by minimizing the energy difference $L$. The partial differentials of the energy difference are now taken with respect to the assumed modal shape constants $E, F$, and $G$, i.e.:

$$
\partial L / \partial E_{k \ell}=0 ; \quad \partial L / F_{k \ell}=0 ; \quad \partial L / \partial G_{k \ell}=0
$$

where $k$ and $\ell$ represent the desired numbers of terms taken in the series appearing in (10). In this investigation three terms in $\alpha$ and three terms in $\beta$ were used; thus, $k, \ell=1,2,3$.

With Equations (12), the stiffness and inertia matrices may be obtained. For each equation the assumed modal shape coefficients are collected and separated into the following matrix form:

$$
[S]-\lambda[I]=0
$$

where $[S]$ is the stiffness matrix, $[I]$ is the inertia matrix, and $\lambda=\rho h \omega^{2}$.

This gives a $27 \times 27$ stiffness matrix. The inertia matrix is composed of three submatrices appearing on the diagonal only, with each submatrix being a 3 x 3 matrix.

Having the stiffness and inertia matrices formed, the analysis reduces to a standard eigenvalue problem. Since the Rayleigh-Ritz method was used, the stiffness and inertia matrices are both symmetric. Therefore, the eigenvalue problem is simplified somewhat and there are available computer subroutines to obtain the eigenvalues $\lambda$.

\section{APPLICATION TO FULLY CLAMPED PLATES}

In applying the Rayleigh-Ritz method, the approximate modal shapes assumed must satisfy all of the kinematic boundary conditions. For a plate 
having all of its edges clamped, the boundary conditions are all kinematic and can be expressed mathematically as follows:

$$
\begin{aligned}
& u=v=0 \quad \text { at } \quad x=0, a \text { and } y=0, b \\
& w=\partial w / \partial x=0 \quad \text { at } \quad x=0, a \\
& w=\partial w / \partial y=0 \quad \text { at } \quad y=0, b
\end{aligned}
$$

The approximate assumed modal functions selected to permit (10) to satisfy (14) are as follows:

$$
\begin{aligned}
& \phi_{u n}=\sin 2 \pi n \alpha ; \quad \phi_{u m}=\sin 2 \pi m \beta \\
& \phi_{v n}=\sin 2 \pi n \alpha ; \quad \phi_{v m}=\sin 2 \rho m \beta \\
& \phi_{w n}=\cosh K_{n} \alpha-\cos K_{n} \alpha-C_{n}\left(\sinh K_{n} \alpha-\sin K_{n} \alpha\right) \\
& \phi_{w m}=\cosh K_{m} \beta-\cos K_{m} \beta-C_{m}\left(\sinh K_{m} \beta-\sin K_{m} \beta\right)
\end{aligned}
$$

where $K_{n}, K_{m}$ are clamped-beam function coefficients tabulated in [6].

The presence of the factor 2 in the arguments of $\phi_{u n}, \phi_{u m}, \phi_{v n}, \phi_{v m}$ needs some explanation. If this factor had been omitted, there would be net in-plane motion of the center of the plate for all odd values of $n$ and $m$. Such motion would not be consistent with the symmetry of the normaldeflection modal shape and the boundary conditions; thus, the factor of 2 was used to eliminate the inconsistency. In [13], the factor of 2 was omitted, and it was found that the differences between the results obtained there and the present results were negligible.

\section{NUMERICAL RESULTS}

A computer program was written in FORTRAN IV language to solve (13) using modal functions (15). The program is documented in [13].

Computations were carried out on an IBM System 360, Series 40 computer for a series of rectangular plates clamped to dimensions $a=9 \mathrm{in}$. and $b=6 \mathrm{in}$. and laminated of glass-fiber-reinforced plastic (GFRP). The material properties used for each layer are listed in Table 1. The calcu-

Table 1. Properties for a Single Ply of GFR*

\begin{tabular}{ll}
\hline \multicolumn{1}{c}{ Quantity } & \multicolumn{1}{c}{ Value } \\
\hline Major Young's modulus & $2.695 \times 10^{6} \mathrm{psi}$ \\
Minor Young's modulus & $2.56 \times 10^{6} \mathrm{psi}$ \\
Major Poisson's ratio & 0.242 \\
Minor Poisson's ratio & 0.230 \\
Shear modulus & $0.6 \times 10^{6} \mathrm{psi} * *$ \\
Density & $0.000197 \mathrm{lb}-\mathrm{sec}^{2} / \mathrm{in}^{4}$ \\
Thickness & $0.0105 \mathrm{in}$. \\
*Style 909 fabric consisting of E glass fibers with S-920 finish, impregnated with 828 epoxy resin cured with curing \\
agent Z.
\end{tabular}


lated natural frequencies for the first three axial and transverse modes are listed in Table 2 for four different lamination arrangements: the first is specially orthotropic, the second is generally orthotropic (i.e. plane anisotropic), the third is layered specially orthotropic, and the fourth is layered generally orthotropic (i.e. layered anisotropic).

Table 2. Resonant Frequencies in Hertz (Experimental Values in Parentheses)

Two-Layer, Parallel-Ply Oriented at $0^{\circ}$

\begin{tabular}{|c|ccc|}
\hline $\boldsymbol{m}$ & $\mathbf{1}$ & $\mathbf{2}$ & $\mathbf{3}$ \\
\hline 1 & $101(112)$ & 156 & 244 \\
2 & 230 & 288 & 372 \\
3 & $425(456)$ & 480 & 563 \\
\hline
\end{tabular}

Two-Layer, Parallel-Ply Oriented at $45^{\circ}$

\begin{tabular}{|c|ccc|}
\hline $\boldsymbol{m}$ & $\mathbf{I}$ & $\mathbf{2}$ & $\mathbf{3}$ \\
\hline 1 & $95(104)$ & 145 & 225 \\
2 & 223 & 267 & 344 \\
3 & $415(427)$ & 460 & 527 \\
\hline
\end{tabular}

Four-Layer, Cross-Ply, Top Two Layers at $0^{\circ}$ \& Bottom Two at $90^{\circ}$

\begin{tabular}{|c|ccr|}
\hline $\boldsymbol{n}$ & $\mathbf{1}$ & $\mathbf{2}$ & \multicolumn{3}{c|}{} \\
\hline 1 & $190(193)$ & 300 & 460 \\
2 & 442 & 550 & 710 \\
3 & $820(860)$ & 925 & 1080 \\
\hline
\end{tabular}

Four-Layer, Cross-Ply, Top Two Layers af $45^{\circ}$ \& Botfom Two af $-45^{\circ}$

\begin{tabular}{|c|ccc|}
\hline $\boldsymbol{m}$ & $\mathbf{1}$ & $\mathbf{2}$ & $\mathbf{3}$ \\
\hline 1 & $180(167)$ & 280 & 430 \\
2 & 425 & 512 & 660 \\
3 & $795(860)$ & 875 & 1005 \\
\hline
\end{tabular}




\section{COMPARISON WITH EXPERIMENTAL RESULTS}

The numbers in parentheses in Table 2 are the corresponding resonant frequencies measured experimentally. The plates were clamped to a rigid clamping fixture which was base excited by an electrodynamic shaker. Resonance was determined by the peak amplitude response of a small metallic-foil strain gage at the plate center. Considerable nonlinearity, i.e. increasing frequency with increasing excitation or response amplitude, was observed. Details of the experiments, as well as an approximate, nonlinear, specially orthotropic analysis, are presented in Reference [14]. The resonant frequencies listed in Table 2 were determined by extrapolating the experimental frequency versus amplitude curves to zero amplitude.

It is noted that the agreement between the present calculations and the experimental results was quite good for the lowest mode $(n=1$, $m=1$ ) but only fair for the third longitudinal mode $(n=3, m=1)$. It was somewhat surprising that the calculated results were generally lower than the experimental ones, since the Rayleigh-Ritz method should give an upper bound. Also transverse shear flexibility, interlaminar shear flexibility, and rotatory inertia have all been neglected in the analysis and they would all tend to lower the frequency. However, it is believed that the discrepancy can be attributed to the low value estimated for the shear modulus and used in the calculations.

The plates used in the experiments exhibited some initial curvature of the cylindrical type (i.e. zero Gaussian curvature). However, in all cases, the initial curvatures were uniform, i.e. they had a constant radius of curvature. This would be expected, since this curvature was undoubtedly temperature induced. To investigate analytically the effect of the uniformly-distributed initial bending stresses (induced by flattening the plates in the clamping fixture) on the natural frequencies, this effect was added to the Rayleigh-Ritz analysis using the same clamped-clamped beam functions as in the analysis already described. It was found that under these conditions (fully clamped edges), the initial curvature had no effect on the natural frequencies.

It should be mentioned that the use of a more highly anisotropic composite material, such as boron or graphite reinforced composites, would have demonstrated the bending-stretching coupling effect more dramatically, as suggested in a recent paper by Whitney [15].

\section{CONCLUSIONS}

The Rayleigh-Ritz free vibrational analysis presented here is believed to be sufficiently accurate for most engineering purposes for calculation of 
the natural frequencies of clamped rectangular panels consisting of arbitrarily laminated, thin, anisotropic layers.

\section{APPENDIX A \\ EVALUATION OF THE MIXED \\ TRIGONOMETRIC-CHARACTERISTIC BEAM FUNCTION INTEGRALS}

This appendix is included for future solutions of the Rayleigh-Ritz formulation for fully clamped rectangular plates, where the characteristic beam function [6] for a fully clamped beam is used as the assumed modal function for the normal deflection and simple trigonometric functions are used for the tangential displacements; see Equations (15). When a simple trigonometric function and the clamped beam function are used as assumed modal functions, the Rayleigh-Ritz solution yields trigonometric-trigonometric, trigonometric-beam, and beam-beam combinations of integrals. Trigonometric-trigonometric integrals are evaluated in standard integral tables and the beam-beam type integrals were given by Felgar [16]. However, for the combination of trigonometric and beam type integrals, no published information could be found. Therefore, these integrals were evaluated and are listed below, using the following notation:

$\phi_{u n}=$ trigonometric function of argument $2 n \pi ; \phi_{u n}=$ characteristic clamped beam function of argument $K_{n} ; C_{n}=$ coefficient tabulated in [6].

$$
\begin{aligned}
& \frac{\int_{0}^{1} \phi_{u n} \phi_{w n} d \alpha:}{} \\
& \quad \frac{1}{K_{n}^{2}+(2 n \pi)^{2}}\left\{K_{n} \sinh K_{n} \sin 2 n \pi-2 n \pi \cosh K_{n} \cos 2 n \pi+2 n \pi\right\} \\
& \quad+\left\{\frac{\cos \left(2 n \pi+K_{n}\right)-1}{2\left(2 n \pi+K_{n}\right)}+\frac{\cos \left(2 n \pi-K_{n}\right)-1}{2\left(2 n \pi-K_{n}\right)}\right\} \\
& \quad-\frac{C_{n}}{(2 n \pi)^{2}+K_{n}^{2}}\left\{K_{n} \cosh K_{n} \sin 2 n \pi-2 n \pi \sinh K_{n} \cos 2 n \pi\right\} \\
& \quad+C_{n}\left\{\frac{\sin \left(2 n \pi-K_{n}\right)}{2\left(2 n \pi-K_{n}\right)}-\frac{\sin \left(2 n \pi+K_{n}\right)}{2\left(2 n \pi+K_{n}\right)}\right\} \\
& \int_{0}^{1} \phi_{u n} \phi_{w n}^{\prime} d \alpha: \\
& \quad \frac{K_{n}}{(2 n \pi)^{2}+K_{n}^{2}}\left\{K_{n} \cosh K_{n} \sin 2 n \pi-2 n \pi \sinh K_{n} \cos 2 n \pi\right\} \\
& \quad+K_{n}\left\{\frac{\sin \left(2 n \pi-K_{n}\right)}{2\left(2 n \pi-K_{n}\right)}-\frac{\sin \left(2 n \pi+K_{n}\right)}{2\left(2 n \pi+K_{n}\right)}\right\} \\
& \quad-\frac{C_{n} K_{n}}{K_{n}^{2}+(2 n \pi)^{2}}\left\{K_{n} \sinh K_{n} \sin 2 n \pi-2 n \pi \cosh K_{n} \cos 2 n \pi+2 n \pi\right\} \\
& \quad-C_{n} K_{n}\left\{\frac{\cos \left(2 n \pi+K_{n}\right)-1}{2\left(2 n \pi+K_{n}\right)}-\frac{\cos \left(2 n \pi-K_{n}\right)-1}{2\left(2 n \pi-K_{n}\right)}\right\}
\end{aligned}
$$


Free Vibrations of Unsymmetrically Laminated Anisotropic Plates

$\underline{\int_{0}^{1} \phi_{u n} \phi_{w n}^{\prime \prime} d \alpha}$

$\frac{K_{n}^{2}}{K_{n}^{2}+(2 n \pi)^{2}}\left\{K_{n} \sinh K_{n} \sin 2 n \pi-2 n \pi \cosh K_{n} \cos 2 n \pi+2 n \pi\right\}$

$-K_{n}^{2}\left\{\frac{\cos \left(2 n \pi+K_{n}\right)-1}{2\left(2 n \pi+K_{n}\right)}+\frac{\cos \left(2 n \pi-K_{n}\right)-1}{2\left(2 n \pi-K_{n}\right)}\right\}$

$+\frac{C_{n} K_{n}^{2}}{K_{n}^{2}+(2 n \pi)^{2}}\left\{K_{n} \cosh K_{n} \sin 2 n \pi-2 n \pi \sinh K_{n} \cos 2 n \pi\right\}$

$-C_{n} K_{n}^{2}\left\{\frac{\sin \left(2 n \pi-K_{n}\right)}{2\left(2 n \pi-K_{n}\right)}-\frac{\sin \left(2 n \pi+K_{n}\right)}{2\left(2 n \pi+K_{n}\right)}\right\}$

$\int_{0}^{1} \phi_{u n}^{\prime} \phi_{w n} d \alpha:$

$\frac{2 n \pi}{(2 n \pi)^{2}+K_{n}^{2}}\left\{K_{n} \sinh K_{n} \cos 2 n \pi+2 n \pi \cosh K_{n} \sin 2 n \pi\right\}$

$-2 n \pi\left\{\frac{\sin \left(2 n \pi+K_{n}\right)}{2\left(2 n \pi+K_{n}\right)}+\frac{\sin \left(2 n \pi-K_{n}\right)}{2\left(2 n \pi-K_{n}\right)}\right\}$

$-\frac{2 n \pi C_{n}}{(2 n \pi)^{2}+K_{n}^{2}}\left\{K_{n} \cosh K_{n} \cos 2 n \pi+2 n \pi \sinh K_{n} \sin 2 n \pi-K_{n}\right\}$

$-2 n \pi C_{n}\left\{\frac{\cos \left(K_{n}+2 n \pi\right)-1}{2\left(K_{n}+2 n \pi\right)}+\frac{\cos \left(K_{n}-2 n \pi\right)-1}{2\left(K_{n}-2 n \pi\right)}\right\}$

$\underline{\int_{0}^{1} \phi_{u n}^{\prime} \phi_{u n}^{\prime} d \alpha:}$

$\frac{2 n \pi K_{n}}{(2 n \pi)^{2}+K_{n}^{2}}\left\{K_{n} \cosh K_{n} \cos 2 n \pi+2 n \pi \sinh K_{n} \sin 2 n \pi-K_{n}\right\}$

$-2 n \pi K_{n}\left\{\frac{\cos \left(K_{n}+2 n \pi\right)-1}{2\left(K_{n}+2 n \pi\right)}+\frac{\cos \left(K_{n}-2 n \pi\right)-1}{2\left(K_{n}-2 n \pi\right)}\right\}$

$-\frac{2 n \pi C_{n} K_{n}}{K_{n}^{2}+(2 n \pi)^{2}}\left\{K_{n} \sinh K_{n} \cos 2 n \pi+2 n \pi \cosh K_{n} \sin 2 n \pi\right\}$

$+2 n \pi C_{n} K_{n}\left\{\frac{\sin \left(2 n \pi+K_{n}\right)}{2\left(2 n \pi+K_{n}\right)}+\frac{\sin \left(2 n \pi-K_{n}\right)}{2\left(2 n \pi-K_{n}\right)}\right\}$

$\int_{0}^{1} \phi_{u n}^{\prime} \phi_{w n}^{\prime \prime} d \alpha:$

$\frac{2 n \pi K_{n}^{2}}{K_{n}^{2}+(2 n \pi)^{2}}\left\{K_{n} \sinh K_{n} \cos 2 n \pi+2 n \pi \cosh K_{n} \sin 2 n \pi\right\}$

$+2 n \pi K_{n}^{2}\left\{\frac{\sin \left(2 n \pi+K_{n}\right)}{2\left(2 n \pi+K_{n}\right)}+\frac{\sin \left(2 n \pi-K_{n}\right)}{2\left(2 n \pi-K_{n}\right)}\right\}$

$-\frac{2 n \pi C_{n} K_{n}^{2}}{(2 n \pi)^{2}+K_{n}^{2}}\left\{K_{n} \cosh K_{n} \cos 2 n \pi+2 n \pi \sinh K_{n} \sin 2 n \pi-K_{n}\right\}$

$+2 n \pi C_{n} K_{n}^{2}\left\{\frac{\cos \left(K_{n}+2 n \pi\right)-1}{2\left(K_{n}+2 n \pi\right)}+\frac{\cos \left(K_{n}-2 n \pi\right)-1}{2\left(K_{n}-2 n \pi\right)}\right\}$ 


\section{W. Bert and B. L. Mayberry}

\section{NOMENCLATURE}

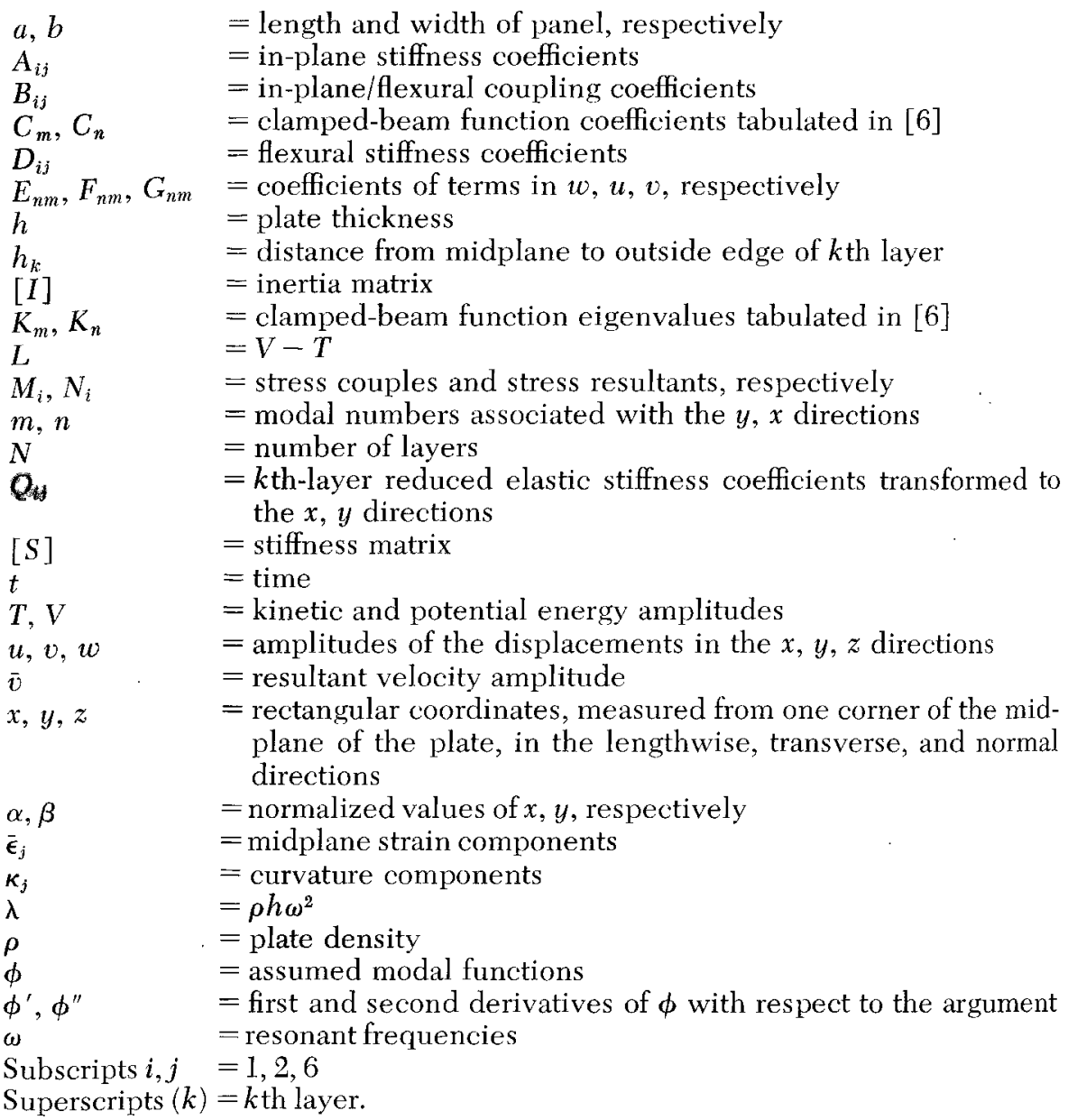

\section{REFERENCES}

1. A. W. Leissa, "Free Vibrations of Elastic Plates", AIAA 7th Aerospace Sciences Meeting, New York, Jan. 20-22, 1969, Paper No. 69-24.

2. K. T. Sundara Raja Tyengar and K. S. Jagadish, "Vibration of Rectangular Orthotropic Plates", Applied Scientific Research, Vol. 13A (1964), p. 37.

3. M. E. Waddoups, "The Vibration Response of Laminated Orthotropic Plates", unpublished M. S. Thesis, Brigham Young University (Aug. 1965).

4. J. E. Ashton, "Anisotropic Plate Analysis", General Dynamics Corp., Fort Worth Div., Contract No. AF 33(615)-5257, Report FZM-4899 (Oct. 12, 1967).

5. J. E. Ashton and M. E. Waddoups, "Analysis of Anisotropic Plates", J. Composite Materials, Vol. 3 (1969), p. 148.

6. D. Young and R. P. Felgar, Jr., "Table of Characteristic Functions Representing Normal Modes of Vibration of a Beam", University of Texas, Austin, Bureau of Engineering Research, Bulletin No. 4913 (July 1949). 


\section{Free Vibrations of Unsymmetrically Laminated Anisotropic Plates}

7. J. E. Ashton and J. D. Anderson, "Dynamic Response of Anisotropic Plates", General Dynamics Corp., Fort Worth Div., Contract No. AF 33(615)-5257, Report FZM-5088 (Mar. 29, 1968).

8. K. S. Pister, "Flexural Vibration of Thin Laminated Plates", Jour. Acoust. Soc. of America, Vol. 31 (1959) , p. 233.

9. N. Ren and Y.-Y. Yu, "Flexural and Extensional Vibrations of Two-Layered Plates", AIAA Journal, Vol. 5 (1967), p. 797.

10. Y. Stavsky, "Thermoelastic Vibrations of Heterogeneous Membranes and Inextensional Plates", AIAA Journal, Vol. 1 (1963), p. 722.

11. Y. Stavsky, "Bending and Stretching of Laminated Aeolotropic Plates", Proc. ASCE, Vol. 87, No. EM6 (Dec. 1961), p. 31.

12. S. W. Tsai, "Mechanics of Composite Materials. Part II-Theoretical Aspects", Air Force Materials Laboratory, AFML-TR-66-149, Part II (Nov. 1966).

13. B. L. Mayberry, "Vibration of Layered Anisotropic Panels", unpublished Master of Engineering (Aerospace) Thesis, University of Oklahoma (June 1968).

14. B. L. Mayberry and C. W. Bert, "Experimental Investigation of Nonlinear Vibrations of Laminated Anisotropic Panels", Bulletin of the 39th Shock and Vibration Symposium, Department of Defense, Monterey, Calif. (Oct. 22-24, 1968).

15. J. M. Whitney, "Bending-Extensional Coupling in Laminated Plates Under Transverse Loading", J. Composite Materials, Vol. 3 (1969), p. 20.

16. R. P. Felgar, Jr., "Formulas for Integrals Containing Characteristic Functions of a Vibrating Beam", University of Texas, Austin, Bureau of Engineering Research, Circular No. 14 (1950). 